\title{
Rowland Clark and Dan Holdeman Site Human Skeletal Remains
}

Carol J. Loveland

Unknown

Follow this and additional works at: https://scholarworks.sfasu.edu/ita

Part of the American Material Culture Commons, Archaeological Anthropology Commons, Environmental Studies Commons, Other American Studies Commons, Other Arts and Humanities Commons, Other History of Art, Architecture, and Archaeology Commons, and the United States History Commons

Tell us how this article helped you.

This Article is brought to you for free and open access by the Center for Regional Heritage Research at SFA ScholarWorks. It has been accepted for inclusion in Index of Texas Archaeology: Open Access Gray Literature from the Lone Star State by an authorized editor of SFA ScholarWorks. For more information, please contact cdsscholarworks@sfasu.edu. 


\section{Rowland Clark and Dan Holdeman Site Human Skeletal Remains}

Creative Commons License

(c) (i) (8)

This work is licensed under a Creative Commons Attribution-NonCommercial 4.0 International License 


\title{
Appendix 2
}

\section{Rowland Clark and Dan Holdeman Site}

\author{
Human Skeletal Remains
}

\author{
Carol J. Loveland
}

\section{INTRODUCTION}

The Rowland Clark site was occupied by Caddoan Indian groups from approximately A.D. 1300-1600+. Twenty one of the 39 burials recovered during the Museum of the Red River excavations were assigned to the earliest McCurtain phase occupation (ca. A.D. 1300-1450); 14 burials were ascribed to a later McCurtain occupation between ca. A.D. 1450 and 1600; four burials belonged to the final McCurtain occupation (ca. A.D. 1600+) of the site. Since infants and children were buried under house floors rather than in the cemetery area associated with each time period, their interment does not necessarily follow the assigned time period. Due to poor preservation and small sample sizes all burials were evaluated as a single Caddoan population.

The burials from the Dan Holdeman site were found in a mound and three cemetery areas located on a terrace adjacent to the Red River. Skeletons of 26 individuals were recovered. The remains of an additional 15 individuals could not be retrieved due to their poor preservation. The acidity of the soil at the site contributed to considerable disintegration of the bones, leaving all burials in fragmentary condition.

Three time periods are represented in the burials from the Dan Holdeman site. Burials 22, 23, and 25 were associated with a Formative Caddoan occupation (that Perino designated the Spiro Focus) dating about A.D. 1000. Interments corresponding with the Middle Caddoan Sanders Focus, dated about A.D. 1200, include Burials 1-20 and 24. One subadult, Burial 21, dates to the latter portion of the McCurtain phase (ca. A.D. 1650). The skeletal material that could not be retrieved represented individuals living during the Formative and Middle Caddoan occupations.

Since much of the data on the osteoarcheology of the Clark and Holdeman sites has been presented in Loveland (1987), specifically stature estimates, skeletal anomalies, and caries rates, it is the purpose of this appendix to summarize other aspects of the skeletal biology of the prehistoric inhabitants of the two sites. However, the poor condition of the skeletons recovered from the sites precludes accurately assessing the biological condition and adaptive efficiency of the people who lived at the site. However, the analyses presented here, and in Loveland (1987), present data that provides a fairly complete picture of Caddoan adaptive efficiency on the Red River in Northeast Texas.

\section{SKELETAL INVENTORIES}

The skeletal remains from the Rowland Clark and Dan Holdeman sites represent Caddoan individuals who lived over a span of several hundred years. Ideally, those burials associated with each site occupation should be treated as a separate sample; however, due to the fragmentary nature of the remains and the small number of burials available for study, the interments from each site were analyzed as single groups. Tables 1 and 2 present a brief description of each burial from the two sites, including sex, age, skeletal elements present, and skeletal anomalies noted. Sex designations in these tables include those 
individuals classified as probable males or females. Hereafter, those burials will be grouped with their respective sex.

Dentition

Dental pathologies occurred commonly in the Rowland Clark population; those observed included caries, broken teeth associated with caries, and abscesses. Table 3 shows the distribution of these dental problems by tooth type and sex. The dentition of several burials was excluded from this analysis since the teeth and alveolar bone were too fragmentary and broken to permit determination of tooth side and position.

The males exhibited slightly more dental problems than the females: two more caries, one more abscess, and 11 more teeth lost antemortem. This probably reflects their greater longevity rather than any difference in the use or care of their teeth.

The dentition of six males and three females at Dan Holdeman was sufficiently complete to determine the condition of the teeth based upon type, position, and side. Table 4 presents the distribution of caries, abscesses, and broken teeth among the 229 teeth examined from these nine individuals. Caries were entirely limited to the posterior dentition; however, it is possible that caries may have occurred in some of the missing anterior teeth.

\section{Cranial Measurements}

Few of the Holdeman or Rowland Clark burials were complete enough to measure. The measurements that were taken are provided in Tables 5 and 6 for comparison with other Caddoan populations.

\section{CONCLUSION}

Despite the poor preservation of the Clark and Holdeman site burials, the bioarcheological analyses of the skeletons contributes to a better understanding of the prehistoric Caddo Indians and their adaptation to their environment (Loveland 1987). Many questions about the skeletal biology of the Rowland Clark and Holdeman populations remain unanswered because the small sample sizes and fragmentary nature of the remains prohibits a comprehensive analysis. However, analysis of the available skeletal data can contribute to our understanding of the Caddoan people. 
Table 1. Description of the Burials from the Rowland Clark Site.

\begin{tabular}{|c|c|c|c|c|c|}
\hline \multicolumn{6}{|c|}{$\begin{array}{l}\text { Intertients Doted } \\
\text { A.D. } 1300-1450\end{array}$} \\
\hline $\begin{array}{l}\text { Surval } \\
\text { Number }\end{array}$ & See. & Ase & $\begin{array}{l}\text { Steletal Elements } \\
\text { Present }\end{array}$ & $\begin{array}{l}\text { Patnology } \\
\text { Osserved }\end{array}$ & Other \\
\hline$!$ & 5 & $20-30$ & 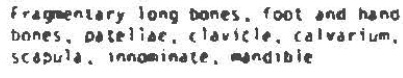 & Mone & \\
\hline$i$ & $\therefore$ & 20.30 & $\begin{array}{l}\text { Elghe licum. irdgrentary sacrum. } \\
\text { ions oones, rdiveriufil }\end{array}$ & None & \\
\hline$=$ & $m$ & $2 \dot{w}-30$ & $\begin{array}{l}\text { Eragmentary bones of mast of } \\
\text { sheleton incluaing andible out } \\
\text { idivarium assing }\end{array}$ & $\begin{array}{l}\text { Dstecdrthritis } \\
\text { distal humeri }\end{array}$ & \\
\hline$\therefore$ & $\therefore$ & $20-3 r_{j}$ & $\begin{array}{l}\text { Eragmentary mondible, colivarsum, } \\
\text { Ever cervica! vertetorat. lony } \\
\text { tones }\end{array}$ & None & \\
\hline 5 & -. & infon: & Fragmentary calvarium & None & \\
\hline$\leq$ & -. & $\begin{array}{l}\text { Sudaduit } \\
\text { iedrly ic } \\
\text { mioteen) }\end{array}$ & 3 moldes (one deciduaus) & None & \\
\hline$\cdot$ & $\vdots$ & 30. & $\begin{array}{l}\text { Frapmentary calvarium. mandible. } \\
\text { long bones. vertebrae }\end{array}$ & & $\begin{array}{l}\text { Rodent gndwing on } \\
\text { long bone tragments }\end{array}$ \\
\hline E & $>$ & $20-30$ & $\begin{array}{l}\text { Fragentary calvarium, andible, } \\
\text { vereabrae, distal humerus }\end{array}$ & $\begin{array}{l}\text { Scheorl's Node. } \\
\text { thoracic vertebrde }\end{array}$ & \\
\hline 5 & -. & Subadule & 2 cermenent moler crowns & mone & \\
\hline
\end{tabular}

\begin{tabular}{|c|c|c|c|c|c|}
\hline & & & $\begin{array}{c}\text { inzemments Doteo } \\
4.0 .1300-1450\end{array}$ & & \\
\hline $\begin{array}{l}\text { Euriel } \\
\text { Numoer }\end{array}$ & sen: & Age & $\begin{array}{l}\text { Skeletal Elements } \\
\text { Present }\end{array}$ & $\begin{array}{l}\text { Pathology } \\
\text { Ooserved }\end{array}$ & Other \\
\hline 10 & 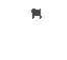 & 30. & $\begin{array}{l}\text { Fragmentary calvarím, mandible. } \\
\text { first and secono cervical vertebrae }\end{array}$ & None & \\
\hline 11 & -. & $\begin{array}{l}\text { Subdoult } \\
\text { (early teeo) }\end{array}$ & fragentery calvarium and mandible & mone & \\
\hline$: 2$ & ? & 30. & $\begin{array}{l}\text { Fragentery andible, calvariun, } \\
\text { long bones }\end{array}$ & None & $\begin{array}{l}\text { Rodent gnawing } \\
\text { on frontal }\end{array}$ \\
\hline$: 3$ & $?$ & 30. & Iragentary calvartus, wandible & Mone & \\
\hline is & ? ? & $20-30$ & Mondible; fragentary calvarium & Mone & \\
\hline$\because \leq$ & $?$ & 30. & $\begin{array}{l}\text { iragmentary calvarium, nasolble. } \\
\text { udper cervical vertebrae }\end{array}$ & $\begin{array}{l}\text { Lorge abscess left ml } \\
\text { mondible }\end{array}$ & $\begin{array}{l}\text { Pacemionian } \\
\text { bodies }\end{array}$ \\
\hline 16 & -. & Sutedult & $\begin{array}{l}\text { very fragmentary colvariuni. } \\
\text { teeth }\end{array}$ & None & \\
\hline$\therefore ?$ & , & Adult & $\begin{array}{l}\text { fragentary calvarium. cervical } \\
\text { vertebrac }\end{array}$ & Hone & \\
\hline 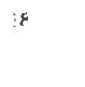 & $k=$ & 30. & $\begin{array}{l}\text { Mondibie: fragoentary calvariua. } \\
\text { cervical vertebrae. feword } \\
\text { shdifs: hand bones }\end{array}$ & $\begin{array}{l}\text { Slight osteophycosis } \\
\text { of cervical verteorde }\end{array}$ & \\
\hline$! 9$ & $?$ & $20-30$ & $\begin{array}{l}\text { Fraquentary calvarium, mandible. } \\
\text { first and second cervical } \\
\text { vertebrae }\end{array}$ & $\begin{array}{l}\text { Mealed depressed } \\
\text { Fracture on oceloitdi }\end{array}$ & home \\
\hline 2: & 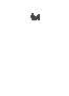 & 30 . & $\begin{array}{l}\text { frayentary calvartum, andiale, } \\
\text { cervical vertebrae, femora shafis }\end{array}$ & $\begin{array}{l}\text { Moderate ostecphylosis } \\
\text { of cervicd vertebrae: } \\
\text { G bution Osteoms on } \\
\text { craniva }\end{array}$ & \\
\hline
\end{tabular}




\section{Table 1, cont}

\begin{tabular}{|c|c|c|c|c|c|}
\hline $\begin{array}{l}\text { Buriai } \\
\text { Number }\end{array}$ & Sex: & age & $\begin{array}{l}\text { Skeletdil clements } \\
\text { Present }\end{array}$ & $\begin{array}{l}\text { Pathology } \\
\text { Observed }\end{array}$ & Ocher \\
\hline \multirow[t]{2}{*}{21} & , & 30. & $\begin{array}{l}\text { Fragmentary andible, calvarium, } \\
\text { lorig banes. foot bones, vertebrae }\end{array}$ & $\begin{array}{c}\text { Periosteal reaction } \\
\text { sibite; osteo- } \\
\text { arthritis distal } \\
\text { femord }\end{array}$ & $\begin{array}{l}\text { Rodent gridwing on } \\
\text { long bones }\end{array}$ \\
\hline & & & $\begin{array}{l}\text { Incerments Dated } \\
\text { A.0. } 1450-1600\end{array}$ & & \\
\hline $\begin{array}{l}\text { Burlad } \\
\text { Number }\end{array}$ & Sex & age & $\begin{array}{l}\text { Steletal clements } \\
\text { Present }\end{array}$ & $\begin{array}{l}\text { Pathology } \\
\text { Observed }\end{array}$ & ozner \\
\hline$\because i$ & ; & $20-30$ & $\begin{array}{l}\text { Fragmentary calwarium, mandible, } \\
\text { first ond second cervical } \\
\text { vertebrae }\end{array}$ & Wone & \\
\hline 23 & -. & Subadult & $\begin{array}{l}\text { Loose deciduous and dermonent } \\
\text { ceeth }\end{array}$ & None & \\
\hline 24 & $s$ & $20-30$ & Mandible: fragenentary colvorium & mone & \\
\hline 25 & M & 30. & $\begin{array}{l}\text { Manoible: frageentary calvartum, } \\
\text { cervical vertebrae }\end{array}$ & $\begin{array}{l}\text { Moderate osteophytos is } \\
\text { of cervical vertebrae }\end{array}$ & $\begin{array}{l}\text { Cremated cranial } \\
\text { irroments included } \\
\text { with buriel }\end{array}$ \\
\hline 26 & $f$ & $20-30$ & $\begin{array}{l}\text { Mundible: fragesntary catvariven, } \\
\text { long bones. ribs, vertebrae. } \\
\text { claricle. hand bones }\end{array}$ & $\begin{array}{l}\text { Slight cribre } \\
\text { otbitalis doth orbits: } \\
\text { periosteal reaction } \\
\text { tibid and fibula }\end{array}$ & $\begin{array}{l}\text { Pacchionian oodies: } \\
\text { rodent gndwing }\end{array}$ \\
\hline 27 & ? & $30+?$ & $\begin{array}{l}\text { few fragents of calvarium and } \\
\text { long boaes }\end{array}$ & Mone & \\
\hline 28 & F & $20-36$ & $\begin{array}{l}\text { Sragmentary calvarium, cervical } \\
\text { vercedrae; mandible }\end{array}$ & $\begin{array}{l}\text { Right mostoid considerat } \\
\text { smaller than left }\end{array}$ & ably \\
\hline 25 & $\mu$ & 30 . & $\begin{array}{l}\text { mandidele: rragmentary calvarivem. } \\
\text { lorg bones, verlebrae }\end{array}$ & $\begin{array}{l}\text { Periostitis right distd } \\
\text { femur shaft; osteoshyzo } \\
\text { cervical vertebree }\end{array}$ & $\begin{array}{l}\text { of } \\
\text { os is }\end{array}$ \\
\hline $3 \mathrm{C}$ & .. & intont & $\begin{array}{l}\text { Right lliuen, loia; left ferur. } \\
\text { rib fragmenis }\end{array}$ & None & $\begin{array}{l}\text { Rooent gnewing } \\
\text { on remur }\end{array}$ \\
\hline $3 !$ & , & Mduli & $\begin{array}{l}\text { Frogmentary lomer lims ono } \\
\text { coot bones }\end{array}$ & Mane & \\
\hline 32 & -. & Intont & Fragmentary bones of skeleton & Nane & \\
\hline 33 & -. & $\begin{array}{l}\text { Subadult } \\
\text { (teendger) }\end{array}$ & $\begin{array}{l}\text { Fragentary lower limb and } \\
\text { foot oones }\end{array}$ & None & \\
\hline 34 & -. & $\begin{array}{l}\text { Subadult } \\
(6-12)\end{array}$ & $\begin{array}{l}\text { frogenencary colvarium. mondible. } \\
\text { cervical vertebrae }\end{array}$ & & $\begin{array}{l}3 \text { odult cervical } \\
\text { vertebrae included }\end{array}$ \\
\hline 35 & $\therefore$ & 30 . & $\begin{array}{l}\text { Erogenentary cranium, andiole. } \\
\text { long Dones. Cervical vertebrae }\end{array}$ & Mone & \\
\hline
\end{tabular}

\begin{tabular}{|c|c|c|c|c|c|}
\hline $\begin{array}{l}\text { Burld: } \\
\text { Number }\end{array}$ & Ses & Age & $\begin{array}{l}\text { Skeietol Elements } \\
\text { Dresent }\end{array}$ & $\begin{array}{l}\text { Pathulogy } \\
\text { Ooserved }\end{array}$ & Other \\
\hline \multirow[t]{2}{*}{36} & $\cdots$ & Subadutc & $\begin{array}{l}\text { Loose deciduaus and permanent } \\
\text { teeth }\end{array}$ & Mone & \\
\hline & & & $\begin{array}{l}\text { Interments Doied } \\
\text { A.D. } 1600 .\end{array}$ & & \\
\hline $\begin{array}{l}\text { Auriai } \\
\text { Number }\end{array}$ & Se $x$ & Age & $\begin{array}{l}\text { Skeletal Elements } \\
\text { Present }\end{array}$ & $\begin{array}{l}\text { Pathology } \\
\text { Observed }\end{array}$ & Other \\
\hline$\therefore$ & ים & sū- & $\begin{array}{l}\text { Froçmentary cronium, aundibie. } \\
\text { long bones }\end{array}$ & $\begin{array}{l}\text { Anscess ml } \\
\text { right masitid }\end{array}$ & $\begin{array}{l}\text { Rowent grawing in } \\
\text { long bones }\end{array}$ \\
\hline 38 & $\cdots$ & $69-30$ & $\begin{array}{l}\text { Fragmentary remuins of most } \\
\text { or steititor. }\end{array}$ & tone & \\
\hline :' & $\cdot$. & nisnt & $\begin{array}{l}\text { Irogmeritars calvarium. pas: } \\
\text { cranida: bones }\end{array}$ & None & \\
\hline
\end{tabular}

- Sex designation densica as follows $\quad F$ iemale

F. pribalute temale

1i. - probat

si- probatble male

- sulvaduli no sea

straduli. no sea

vetermination 
Table 2. Description of the Burials from the Dan Holdeman Site.

\begin{tabular}{|c|c|c|c|c|c|c|}
\hline Burial & Number & Sex" & Age & $\begin{array}{l}\text { Skeletal Elerrents } \\
\text { Present }\end{array}$ & $\begin{array}{l}\text { Anorralles } \\
\text { Observed }\end{array}$ & Commen:s \\
\hline & 1 & Al? & $20-10$ & $\begin{array}{l}\text { Fragrentury calverium, manal- } \\
\text { ble. icipula, eloviele. mumerus. } \\
\text { femors }\end{array}$ & None & \\
\hline & $2 A$ & A: & $20 \cdot 30$ & $\begin{array}{l}\text { Ferrora: mandible: frogmentary } \\
\text { renrains of most of skeleton }\end{array}$ & Kyphosis LS-S1 & $\begin{array}{l}\text { Intrusive } \\
\text { Durial: roce-: } \\
\text { gnawing on :en and } \\
\text { and humerus }\end{array}$ \\
\hline & 28 & Ai & 30. & $\begin{array}{l}\text { Frogrentary culvarlum, manoi- } \\
\text { ble, vertebrae, clovicle, libis. } \\
\text { femora, innominate, humeri }\end{array}$ & $\begin{array}{l}\text { Healed depressed } \\
\text { fracture lefe frontal: } \\
\text { periosteal reaction } \\
\text { shaft uft tibia }\end{array}$ & \\
\hline & 3 & $M$ & $20-30$ & $\begin{array}{l}\text { Ferrur; nandible; frogmentary } \\
\text { calvarlum, long bones. foot } \\
\text { bones. clavicle. ribs. patellae. } \\
\text { vertebrae. Innominates }\end{array}$ & $\begin{array}{l}\text { Two button osteorsas } \\
\text { frontal bone }\end{array}$ & \\
\hline & 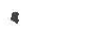 & $m$ & $20-30$ & $\begin{array}{l}\text { Fragmentary Dones of most } \\
\text { of gkeleton }\end{array}$ & None & \\
\hline & 3 & $M ?$ & $20-30$ & $\begin{array}{l}\text { Fragmantary calvarium, randible. } \\
\text { cenvical vertebrae. ulus. } \\
\text { ferrora shafis }\end{array}$ & $\begin{array}{l}\text { Abscess right maxill, } \\
\text { between } M_{1} \text { and } \mu_{2}\end{array}$ & \\
\hline & 6 & $F$ & $20-30$ & $\begin{array}{l}\text { Mandible: fragmentary calvarium, } \\
\text { long bones, innominates, scapulae }\end{array}$ & $\begin{array}{l}\text { Considerable thickening } \\
\text { of partezals slong } \\
\text { ridline }\end{array}$ & \\
\hline
\end{tabular}

\begin{tabular}{|c|c|c|c|c|c|c|}
\hline Burial & Number & $\operatorname{sex}$ & Age & $\begin{array}{l}\text { Skeletal Elerrants } \\
\text { Present }\end{array}$ & $\begin{array}{l}\text { Anoraties } \\
\text { Observed }\end{array}$ & Consenis \\
\hline & 7 & $\boldsymbol{F}$ & 30. & $\begin{array}{l}\text { Tibia: numerus: radius: mandi- } \\
\text { ble; fragmanury long bones. } \\
\text { foot bones. Patella, calvarium }\end{array}$ & None & $\begin{array}{l}\text { Rodent gnawing } \\
\text { on long bores: } \\
\text { remains of iniant } \\
\text { inot recoveree } \\
\text { reported near } \\
\text { right hurerus }\end{array}$ \\
\hline & 8 & 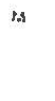 & $20-30$ & $\begin{array}{l}\text { Fragrenlary calvariurr. mandible } \\
\text { long bones. fool bones. Scapula, } \\
\text { ribs, sternarr. }\end{array}$ & $\begin{array}{l}\text { Periosteal reactlon } \\
\text { tibla fragrients: } \\
\text { enarsel nypoplasia } \\
\text { maxillary inclsors }\end{array}$ & \\
\hline & 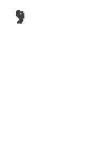 & SA & $12-18$ & $\begin{array}{l}\text { Fragrenumy calvariur., ribs. } \\
\text { vertebrae. Hecrur., foot } \\
\text { bones. ferrora shafis }\end{array}$ & Hone & 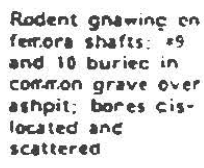 \\
\hline & 10 & $\mathbf{M}^{2}$ & $20-30$ & $\begin{array}{l}\text { Lett feriur: fragrenury long } \\
\text { oones. clavicle, hand bones. } \\
\text { scopula }\end{array}$ & Mone & \\
\hline & 11 & ? & 30. & $\begin{array}{l}\text { Frughentimey calvarium. mandi- } \\
\text { ole. vertabrse }\end{array}$ & Nane & \\
\hline & 12 & $?$ & $20-30$ & $\begin{array}{l}\text { Fragmentary call arlum, randi- } \\
\text { ble. tong bones. ribs. clavi- } \\
\text { cle. vertebrae. Calus }\end{array}$ & $\begin{array}{l}\text { Fiorous oysplesia } \\
\text { on shaft of lang bone }\end{array}$ & $\begin{array}{l}\text { Rodent } \\
\text { gndwing on } \\
\text { !ong cones }\end{array}$ \\
\hline
\end{tabular}


Table 2, cont

\begin{tabular}{|c|c|c|c|c|c|}
\hline Burial Number & sex $x^{\circ}$ & Age & $\begin{array}{l}\text { Skeletal Elerents } \\
\text { Present }\end{array}$ & $\begin{array}{l}\text { Anomalies } \\
\text { Observed }\end{array}$ & Commeris \\
\hline 13 & $M$ & $20-30$ & $\begin{array}{l}\text { Femora: tibia: patellse: } \\
\text { radius: fragrentary remiains } \\
\text { of most of skeleton }\end{array}$ & None & $\begin{array}{l}\text { Burial is tac } \\
\text { intruded uaca } \\
\text { Burial is }\end{array}$ \\
\hline 14 & SA & $2-6$ & $\begin{array}{l}\text { Fragmentary remains of nost } \\
\text { of sheleton }\end{array}$ & $\begin{array}{l}\text { Cribry orditalia } \\
\text { right orbit (left } \\
\text { orbit rissing) }\end{array}$ & \\
\hline 15 & SA & $0=1$ & $\begin{array}{l}\text { Fragrentary renains of nost } \\
\text { of skeleton }\end{array}$ & None & \\
\hline 16 & $F$ & $20-30$ & $\begin{array}{l}\text { Alandible: fragnientary calvar- } \\
\text { ium. long bones }\end{array}$ & $\begin{array}{l}\text { Periosteal reaclion } \\
\text { thiae shatis }\end{array}$ & \\
\hline 17 & F! & 30. & $\begin{array}{l}\text { Fragn:entary calvariun, verte- } \\
\text { orae, randible, long bones. } \\
\text { patella }\end{array}$ & $\begin{array}{l}\text { Button osteora } \\
\text { left frontar }\end{array}$ & \\
\hline 10 & M? & 30. & $\begin{array}{l}\text { Fragrentary calvartur, nandi- } \\
\text { bie, long bones }\end{array}$ & $\begin{array}{l}\text { Temporandibular } \\
\text { joint disease }\end{array}$ & $\begin{array}{l}\text { Large oachor àn } \\
\text { bodies }\end{array}$ \\
\hline 19 & SA & $2-5$ & Fragrentory calvariun: & None & \\
\hline 20 & SA 1 & $12-10$ & $\begin{array}{l}\text { Alendible: fragmentary remains } \\
\text { of r.ost of skaleton }\end{array}$ & $\begin{array}{l}\text { Denign fibrous } \\
\text { corticat defect } \\
\text { on right distal } \\
\text { ienut shaft }\end{array}$ & $\begin{array}{l}\text { Rodent grewing } \\
\text { on long boue } \\
\text { shafts anc t.er- } \\
\text { dible }\end{array}$ \\
\hline 21 & SA & $2-5$ & $\begin{array}{l}\text { Mandible: fragmentary calvar- } \\
\text { ium. long bones. vertebrae. } \\
\text { innoninate. ribs, clavicle }\end{array}$ & $\begin{array}{l}\text { Cribra orbitalia } \\
\text { both orbits: } \\
\text { mild portic nyper- } \\
\text { ostosis frontal }\end{array}$ & \\
\hline
\end{tabular}

\begin{tabular}{|c|c|c|c|c|c|c|}
\hline Burial Number & $\operatorname{sex}$ & Age & $\begin{array}{l}\text { Skeletal } \\
\text { Pres }\end{array}$ & $\begin{array}{l}\text { Elerrents } \\
\text { sent }\end{array}$ & $\begin{array}{l}\text { Anomalies } \\
\text { Observed }\end{array}$ & Commen:s \\
\hline 22 & ? & 30. & $\begin{array}{l}\text { Fragrentary } \\
\text { premolars }\end{array}$ & cavariur., reotars. & Mone & \\
\hline 23 & ? & Actult & $\begin{array}{l}\text { Fragmentory } \\
\text { ble }\end{array}$ & Colvarium, resndi- & None & \\
\hline 28 & SA & $6-12$ & $\begin{array}{l}\text { Pragoentary } \\
\text { Die }\end{array}$ & Caivariurr, mand,- & $\begin{array}{l}\text { Enamel hyDopiasid } \\
\text { centrat maxillery } \\
\text { incisor }\end{array}$ & \\
\hline 25 & SA & $6-12$ & Fragrentary & caivariun & None & \\
\hline - Sex aesugnation & a den & oted as & $\begin{array}{l}F \\
F: \\
M \\
M ! \\
\vdots \\
\text { SA }\end{array}$ & $\begin{array}{l}\text { - Fenale } \\
\text { - Probable Fende } \\
\text { - Male } \\
\text { - Probable Mule } \\
\text { - Sex Undetermined } \\
\text { - Subadult. No Sex }\end{array}$ & eterritnation & \\
\hline
\end{tabular}


Table 3. Frequency of Common Dental Pathologies in the Clark Population.

Males (5)

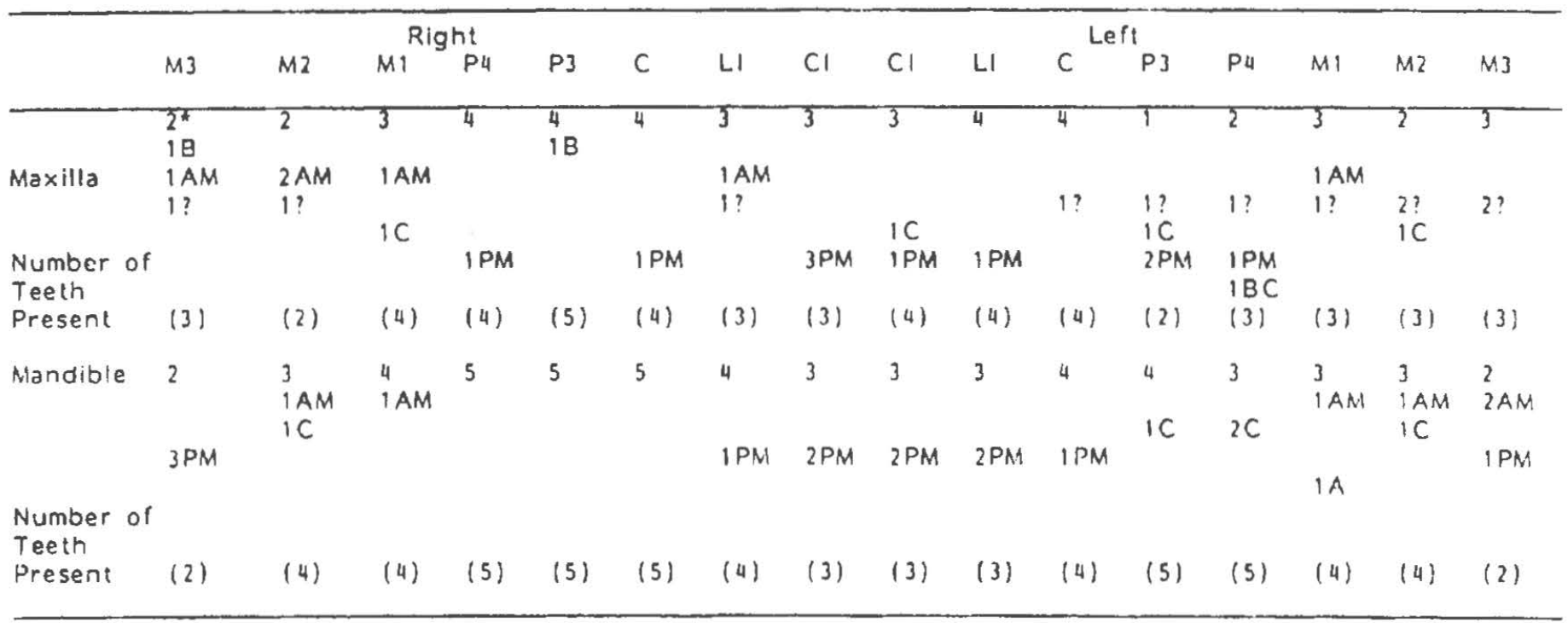

Femoles (6)

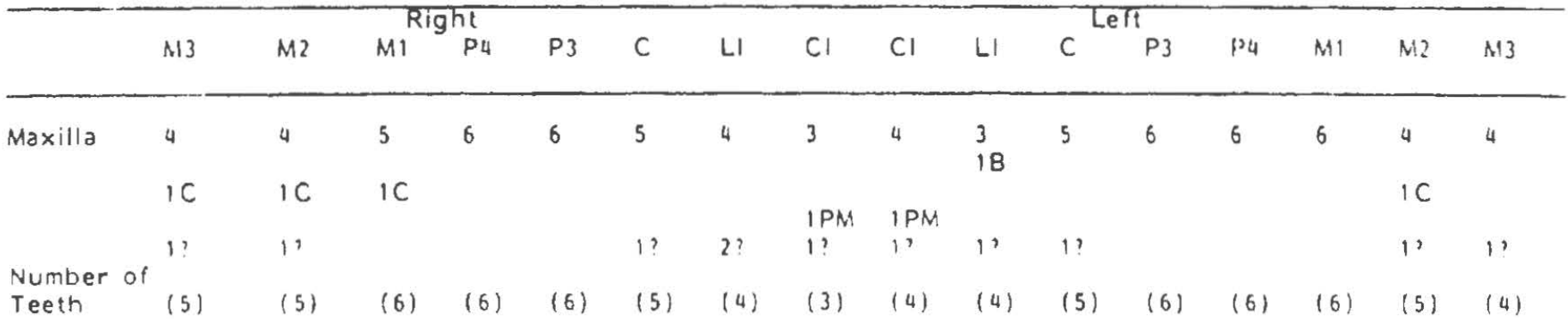


Table 3, cont.

Females (6)

\begin{tabular}{|c|c|c|c|c|c|c|c|c|c|c|c|c|c|c|c|c|}
\hline & \multirow[b]{2}{*}{ M3 } & \multicolumn{4}{|c|}{ Rाght } & \multirow[b]{2}{*}{$c$} & \multirow[b]{2}{*}{$\mathrm{LI}$} & \multirow[b]{2}{*}{$\mathrm{Cl}$} & \multirow[b]{2}{*}{$\mathrm{Cl}$} & \multirow[b]{2}{*}{ LI } & \multicolumn{2}{|c|}{ Left } & \multirow[b]{2}{*}{$\mathrm{Pu}_{4}$} & \multirow[b]{2}{*}{ MI } & \multirow[b]{2}{*}{ M2 } & \multirow[b]{2}{*}{ M3 } \\
\hline & & $M 2$ & M1 & $P_{4}$ & P3 & & & & & & $c$ & $P_{3}$ & & & & \\
\hline \multirow[t]{4}{*}{ Mandible } & 5 & 5 & 5 & 5 & 6 & 5 & 6 & 3 & ${ }_{18}^{4}$ & $\begin{array}{l}5 \\
1 B\end{array}$ & 5 & 6 & 6 & $\begin{array}{l}4 \\
1 B\end{array}$ & 6 & 4 \\
\hline & & & IC & $1 \mathrm{C}$ & & & & & & & & & & & & \\
\hline & & $\mid P M$ & & & & $1 P M$ & & 2PM & IPM & & $1 \mathrm{C}$ & & & $1 \mathrm{C}$ & & \\
\hline & $1 ?$ & & & & & & & 1? & & & & & & & & $\begin{array}{l}1 ? \\
\text { IAM }\end{array}$ \\
\hline $\begin{array}{l}\text { Number of } \\
\text { Teeth } \\
\text { Present }\end{array}$ & (5) & (5) & (6) & $(6)$ & (6) & (5) & (6) & (3) & (5) & (6) & (6) & (6) & $(6)$ & (5) & (6) & (4) \\
\hline
\end{tabular}

Adult, Sex Undetermined

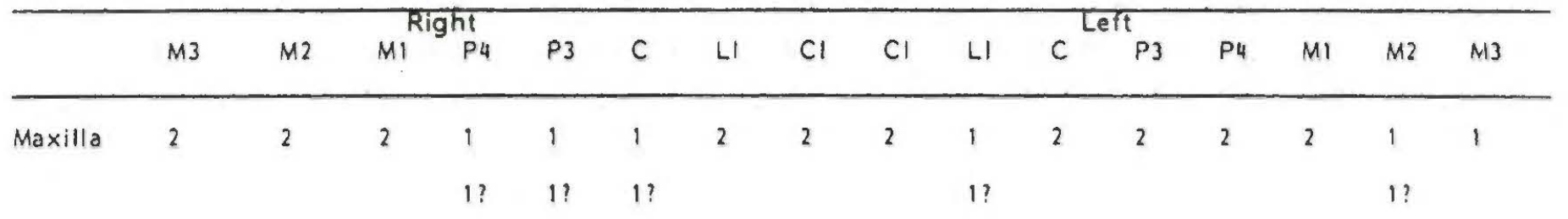

\begin{tabular}{|c|c|c|c|c|c|c|c|c|c|c|c|c|c|c|c|c|}
\hline $\begin{array}{l}\text { Number of } \\
\text { Teeth } \\
\text { Present }\end{array}$ & (2) & (2) & (2) & (1) & (1) & (1) & (2) & (2) & (2) & (1) & (2) & (2) & (2) & (2) & (1) & $\begin{array}{l}\text { IPM } \\
\text { (1) }\end{array}$ \\
\hline Mandible & 2 & 2 & 2 & 2 & 2 & 2 & 2 & 1 & $\begin{array}{l}1 \\
1 ?\end{array}$ & $\begin{array}{l}1 \\
1\end{array}$ & 2 & 2 & 2 & 1 & 2 & 2 \\
\hline $\begin{array}{l}\text { Number of } \\
\text { Teeth } \\
\text { Present }\end{array}$ & (2) & (2) & (2) & (2) & (2) & (2) & (2) & $\begin{array}{l}\text { IPM } \\
\text { (1) }\end{array}$ & (1) & (1) & (2) & (2) & (2) & (2) & (2) & (2) \\
\hline $\begin{array}{l}\text { A - numbe } \\
\text { A - Absce } \\
\text { PM - Postn } \\
B C \text { - Carie }\end{array}$ & $\begin{array}{l}\text { r of } \\
\text { ss } \\
\text { norte } \\
\text { es }\end{array}$ & oss & & & & & $\begin{array}{l}C- \\
A M \\
?- \\
C A\end{array}$ & $\begin{array}{l}\text { one a } \\
\text { Carie }\end{array}$ & id to & $\begin{array}{l}\text { loss } \\
\text { th mi } \\
\text { cess }\end{array}$ & ing & & & & & \\
\hline
\end{tabular}

- Bone and tooth missing

Caries, abscess 
Table 4. Frequency of Dental Lesions in the Holdeman Population.

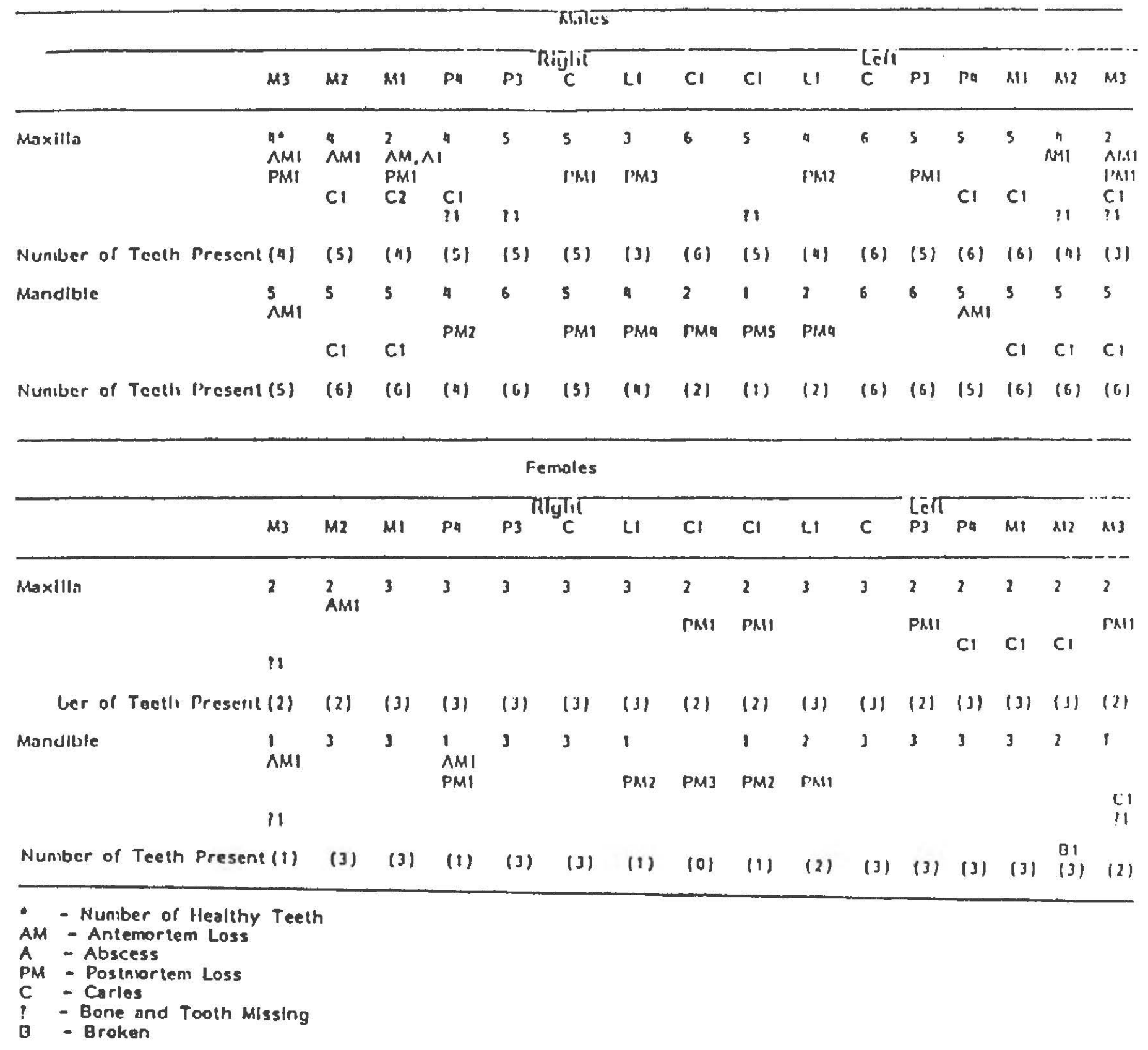


Table 5. Cranial Measurements (mm) of the Clark Interments.

\begin{tabular}{|c|c|c|c|c|}
\hline \multirow[b]{2}{*}{ Measurement } & \multicolumn{2}{|c|}{ Male } & \multicolumn{2}{|c|}{ Female } \\
\hline & $\begin{array}{l}\text { Number } \\
\text { of Cases }\end{array}$ & Mean & $\begin{array}{l}\text { Number } \\
\text { of Cases }\end{array}$ & Mean \\
\hline Maximum Length & 4 & 168.3 & -- & -- \\
\hline Maximum Breadth & 4 & 145.3 & -- & -- \\
\hline Symphysis Height & 5 & 35.8 & 4 & 36.0 \\
\hline Diameter Bigonial & 5 & 104.4 & 4 & 97.5 \\
\hline Diameter Bicondylar & 3 & 124.7 & 3 & 123.0 \\
\hline Height Ascending Ramus & 5 & 64.8 & 4 & 58.3 \\
\hline Corpal Length Gonion-Gnathion & 5 & 89.0 & 4 & 89.0 \\
\hline
\end{tabular}

Table 6. Cranial Measurements (mm) of the Holdeman Interments.

\begin{tabular}{|c|c|c|c|c|}
\hline Measurement & $\begin{array}{l}\text { Male } \\
\text { Number } \\
\text { of Cases }\end{array}$ & Mc:an & $\begin{array}{l}\text { Female } \\
\text { Number } \\
\text { of Cases }\end{array}$ & Me:ill \\
\hline Maximum Length & -- & -- & 2 & 157.0 \\
\hline Maximum Breadth & -- & -- & 2 & 142.0 \\
\hline Minimum Frontal Breadth & -- & -- & 2 & 93.0 \\
\hline Symphysis Height & 1 & 35.0 & 2 & 34.0 \\
\hline Diameter Bigonial & 2 & 105.0 & 1 & 96.0 \\
\hline Diameter Bicondylar & 2 & 131.0 & 1 & 119.0 \\
\hline Height Ascending Ramus & 2 & 58.0 & 2 & 57.0 \\
\hline
\end{tabular}




\section{REFERENCES CITED}

Loveland, Carol J.

1987 Human Skeletal Remains from the Clark and Holdeman Sites, Red River County, Texas. Bulletin of the Texas Archeological Society 57:165-181.

\section{ACKNOWLEDGMENTS}

I wish to thank Gregory Perino for making it possible for me to study these skeletal collections. Dr. John B. Gregg, School of Medicine, the University of South Dakota, Sioux Falls, South Dakota, kindly consented to evaluate two of the skeletal lesions from the Holdeman site. I extend my appreciation to him. 